\title{
Publishing in science: a collaborative process
}

\author{
Hilary P. Grocott, MD
}

Received: 9 September 2014 / Accepted: 17 September 2014/Published online: 25 September 2014

(C) Canadian Anesthesiologists' Society 2014

This editorial marks my first year as Editor-in-Chief (EIC) of the Canadian Journal of Anesthesia, and although it appears here in our December issue, it actually went "to press" in September. By then, I had dedicated more than eight months to reading, reviewing, critiquing, and editing almost 500 submissions to the Journal. While this is early in what I hope will be a long tenure in this post, it is an appropriate time to reflect on what has transpired over these early months and also to convey some thoughts on how to shape our Journal's publishing future.

This has been a momentous year for the Journal - one in which we celebrated our $60^{\text {th }}$ anniversary ${ }^{1}$ and one in which we continued to realize significant growth. This progress is reflected in our growing readership, with the Journal's subscriber base at its largest ever, as well as in our publication's evolving overall "impact" on our field. Our 2013 impact factor - namely, the ratio of the number of articles from the Journal's 2011-2012 contents cited in the 2013 scientific literature compared with the number of articles that we published in that year - is now 2.5, our highest to date.

Although I previously worked on the Journal for many years as a rank and file member of the editorial board, my perspective on scientific publishing has evolved considerably since taking on this new role. I have gained a new appreciation for the tireless efforts of reviewers and board members, as well as editorial and publishing staff, to whom I am enormously thankful for their efforts. Similarly, I also deeply appreciate the efforts of our

H. P. Grocott, MD (ه)

Departments of Anesthesia \& Perioperative Medicine and

Surgery, St. Boniface Hospital, University of Manitoba, CR3008-369, Tache Avenue, Winnipeg, MB R2H 2A6, Canada

e-mail: hgrocott@sbgh.mb.ca authors themselves, whose contributions continue to advance the knowledge and understanding of our field and, along with it, the reputation of the Journal, ever forward.

Authors with firsthand experience can easily appreciate the long and arduous process from manuscript submission to publication. Of course, this initiative begins long before manuscript submission, with the many hundreds of hours spent conceiving, executing, analyzing, considering, and reporting on various research projects or other scholarly activities. The enormous effort that this endeavour requires eventually comes to fruition on the printed page, but those involved at the various stages of this process will undoubtedly attest to the inherent challenges and frequent frustrations. That said, the potential rewards, though often intangible, are too numerous to mention, but ultimately, they are aimed at improving our understanding of science and, importantly, the care of our patients for whom most of us originally entered this field.

Publishing scientific material is never an easy task. Those who have faced the submission, review, and (unfortunately too often) rejection sequence can attest to the frustration that many authors have experienced, myself included. Too often what we consider to be our "best work" is rejected outright during journal review. At other times, a submission is favourably reviewed and advanced for publication, but not until authors have seemingly been made to jump through endless "hoops". In an ideal world, the peer review process of vetting submissions should be an active and constructive collaboration involving the authors, reviewers, editors, and even the readers who importantly provide their own post-publication oversight with frequent discourse in letters of correspondence. Admittedly, the submission process is not always perceived that way. The remainder of this editorial is 
largely directed to prospective authors - it is intended as encouragement and assistance and conveyed in a true collaborative spirit.

I frequently receive inquiries from prospective authors, both novice and seasoned, with the question, "are you interested in publishing this paper?" Before I respond, I usually consider if the manuscript is credible, valid, important, novel - not to mention potentially reproducible - and also if it will be of interest to our readership. With an ever-expanding number of both print and online journals in which anesthesia-related material can be published, authors have an enormous number of options to consider as a forum for their scholarly work. That being said, there are several seemingly basic and obvious principles that can halt the submission process either before it even gets started or shortly after the very first read. These are concepts and details that every author, whether a "first-timer" or a seasoned veteran author, needs to take into account.

The following tenets of scientific writing, though not necessarily intended to be comprehensive, can be considered a guide to begin the submission and review process on a positive note. Firstly, correct spelling and grammar, though not always fundamental to the accuracy and importance of the science being reported, are nonetheless essential. If these fundamentals are poorly addressed, they can be a minor annoyance to the various reviewers, perhaps lessening their enthusiasm for investing their time in the review process. At worst, they could impair the reviewers' ability to understand what should be a clearly transmitted message (i.e., the scientific findings). Both of these factors can adversely affect the reviewing outcome. This issue is easy to address with software and online resources that are widely available to assist authors. ${ }^{\text {A }}$ Indeed, the Journal continues to expand its international scope and encourages and receives many submissions from authors who could make good use of these resources, including those whose first language is not English.

Other readily available tools that should be considered during the writing ${ }^{2}$ but, more importantly, during the earlier planning and execution of studies, are the easily accessible reporting guidelines outlined by the Enhancing the QUAlity and Transparency Of health Research (EQUATOR) network (www.equator-network.org). This international initiative facilitates wide availability of writing guidelines (e.g., the CONSORT guidelines for randomized controlled trials), allowing them to serve as useful "checklists" highlighting the many "must have"

\footnotetext{
A Springer Online Writing Resources. English Language Editing. Available from URL: http://www.springer.com/medicine/anesthesio logy/journal/12630 (accessed September 2014).
}

elements that should be included in every study and, consequently, in every manuscript. Indeed, these design elements are critical for improving scientific communication, which helps to ensure reliability, transparency, accuracy, and, ultimately, the overall value of this communication. The guidelines are also used increasingly by reviewers to identify items that should be included in every paper, with heavy scrutiny placed on their presence or absence. In other words, ensuring that these design elements are explicitly detailed and included in every manuscript is another aspect that facilitates the reviewers' task and, thus, increases the likelihood of acceptance. The importance of using the EQUATOR guidelines long before a manuscript is prepared - i.e., early in the design phase of any study - is underappreciated. This approach helps to ensure that studies are conducted with all the necessary elements for successful publication.

Trial registration on a publically available registration site (such as www.clinicaltrials.gov) is a fundamentally important aspect of planning, executing, and reporting a study. Although this has long been a strongly encouraged suggestion of many journals during their submission process, trial registration will soon be a ubiquitous requirement. The importance of writing and reviewing a paper in the context of its original design (e.g., with $a$ priori defined endpoints) cannot be underestimated. This is important for randomized controlled trials, but also for the various other reports that are submitted, from observational studies through even to case reports.

Statistical issues are a fundamental part of every research report and receive a high degree of attention during the review process. Accordingly, early consultation and acquisition of statistical resources goes a long way to the successful planning, conduct and eventual publication of any study. This facet is too often overlooked, but its importance cannot be overestimated. Many a study report is stopped before it gets past even the first review because of statistical issues. Ideally, this consultation should begin at the earliest stages of study planning, but if not already undertaken, it should, at the very least, be incorporated into the final manuscript writing and author-driven editing. Indeed this is another example of where the EQUATOR guidelines have value, as their SAMPL guideline ${ }^{\mathrm{B}}$ for statistical reporting provides guidance. Many journals have their own preferences or templates for reporting statistics and other related issues, and it would serve prospective authors well to consider these before submission.

\footnotetext{
$\overline{{ }^{B} \text { EQUATOR Network. Basic Statistical Reporting for Articles }}$ Published in Biomedical Journals: the "Statistical Analyses and Methods in the Published Literature" or SAMPL Guidelines. Available from URL: http://www.equator-network.org/reportingguidelines/sampl/ (accessed September 2014).
} 
Indeed, each journal, and ours is no exception, has its own unique overall formatting and guidelines for submission. These guidelines for authors ${ }^{\mathrm{C}}$ are easily accessible online and should be a first-referenced resource and starting point for every prospective author. Though admittedly these guidelines are somewhat dry reading and often contain rather technical writing, they do provide explicit guidance as to the Journal's expectations during the manuscript submission process. It is somewhat surprising then that there is only sporadic adherence to these guidelines. Indeed, the number of times these instructions are accessed and downloaded each year is typically far fewer than the total number of submissions we receive. Adherence to Journal formatting and instructions is also a point that many reviewers consider, as sometimes it can offer interesting tacit insight about a manuscript's history. For example, if a manuscript submitted to Journal $X$ is actually formatted for Journal $Y$, then it is an easy tipoff to reviewers of Journal $X$ that the manuscript may very likely have already been submitted (and rejected) elsewhere, unnecessarily putting the contents of the submission into question very early in the review process - a clear disadvantage to authors.

In summary, when authors adhere to very straightforward recommendations early in the writing and submission process, their manuscript can be in an optimal position for a positive review and corresponding acceptance for publication. It is to authors' advantage to read the instructions for authors, employ a spelling and grammar check and have a native English speaking/reading author review the work, ensure that a statistician has been involved at all stages of the process, employ the widely accepted reporting guidelines, and last, but certainly not least, register the endeavour in a publically available registration site at the outset.

I look forward to continuing to serve as EIC and actively encourage submissions from the clinical and basic sciences that represent wide-ranging types of articles, including reports of original investigations, reviews, and, yes, sometimes even uniquely informative case reports. ${ }^{3}$ My hope is that this editorial will provide some preliminary guidance to prospective authors and also to investigators planning work that they hope will eventually be published. Reviewers, as well, can refer to this article when considering elements to take into account in their own reading. Publication of rigorously reviewed scientific material benefits us all, and when diligently prepared, can represent the best of collaborations between authors with a scientific message and journals with a forum for its

\footnotetext{
$\overline{\mathrm{C}}$ Canadian Journal of Anesthesia Instructions for Authors. Available from URL: http://www.springer.com/medicine/anesthesiology/ journal/12630 (accessed September 2014).
}

dissemination. I hope to remain involved in this rewarding process for much of the foreseeable future.

\section{Publications scientifiques: un travail collaboratif}

Même si cet éditorial marquant ma première année comme rédacteur en chef du Journal canadien d'anesthésie paraît dans ce numéro de décembre, il est parti sous presse en septembre au moment où j'avais passé plus de huit mois à lire, analyser, commenter et éditer près de 500 soumissions qui avaient été envoyées au Journal. Bien que cela soit encore tôt dans ce que j'espère être un long mandat à ce poste, c'est le bon moment de réfléchir sur ce qui ressort de ces premiers mois est aussi de vous communiquer quelques pensées sur l'avenir de la publication dans notre Journal.

Cela a été une année capitale pour le Journal qui nous a donné l'occasion de célébrer son $60^{\mathrm{e}}$ anniversaire, ${ }^{1}$ mais aussi une année au cours de laquelle nous avons continué à voir une croissance significative. Cela se traduit par un nombre croissant de lecteurs avec une base d'abonnés au Journal plus grande que jamais, et aussi par la croissance de notre « impact» global dans notre domaine. En d'autres termes, le rapport entre le nombre d'articles parus en 2011 - 2012 dans le Journal qui ont été cités dans les articles scientifiques de 2013 comparé au nombre d'articles que nous avons publiés dans la même année (c'est-à-dire, notre facteur d'impact 2013) est aujourd'hui de 2.5 , le plus haut à ce jour.

Bien qu'ayant travaillé de nombreuses années avec le Journal comme bien d'autres membres du comité éditorial, mon point de vue sur l'édition scientifique a considérablement évolué depuis que j'occupe cette nouvelle fonction. J'y ai gagné une nouvelle appréciation des efforts inlassables des réviseurs, des membres du comité éditorial ainsi que du personnel éditorial et de publication envers lesquels je suis extrêmement reconnaissant pour leur travail. De même, j'apprécie profondément les efforts de nos auteurs, dont les contributions continues à faire progresser les connaissances dans notre domaine et avec elles, le Journal, toujours plus loin.

Ceux qui en ont acquis l'expérience personnellement peuvent facilement comprendre que le processus allant de l'envoi d'un manuscrit à sa publication est à la fois long et ardu. Cela commence naturellement longtemps avant l'envoi du manuscrit, avec ces centaines d'heures passées à concevoir, exécuter, analyser, envisager et rapporter différents projets de recherche ou d'autres activités académiques. Les efforts énormes que cela nécessite sont 
habituellement invisibles sur la page imprimée, mais ceux qui sont impliqués par les différentes étapes de ce processus pourront facilement attester des défis, et souvent des frustrations, qui lui sont inhérents. Cela dit, les gratifications potentielles - quoique souvent impalpables - sont trop nombreuses pour les mentionner, mais visent finalement à améliorer notre compréhension de la science et, plus encore, les soins aux patients pour lesquels la majorité d'entre nous se sont d'abord engagés dans cette profession.

La publication de matériel scientifique n'est jamais une tâche aisée. Ceux qui ont vécu la séquence de soumission, révision et (malheureusement trop souvent) rejet, peuvent témoigner de la frustration ressentie par de nombreux auteurs dont je fais aussi partie. Trop souvent, ce que nous considérons comme étant notre «meilleur travail» est rejeté d'emblée au cours de sa révision par un journal. À d'autres moments, le processus de révision est favorable et le manuscrit est proposé pour la publication, mais pas avant que les auteurs aient dû franchir ce qui apparaît comme des dédales infinis. Bien que pas toujours perçu de cette façon, dans un monde idéal, le processus de révision par les pairs consistant à filtrer les soumissions devrait être une collaboration active et constructive impliquant les auteurs, les réviseurs, éditeur et même les lecteurs qui, de manière importante, fournissent leur propre avis post publication dans les arguments que l'on voit régulièrement dans le courrier des lecteurs. C'est maintenant aux futurs auteurs que le reste de cet éditorial s'adresse principalement: il se veut encourageant et utile, dans un véritable esprit de collaboration.

Je reçois souvent des demandes d'information de la part d'auteurs potentiels, aussi bien novices qu'expérimentés, avec cette question «êtes-vous intéressés à publier cet article? » Avant de répondre, je réfléchis souvent en silence sur le manuscrit: est-il crédible, valide, important, novateur, sans oublier potentiellement reproductible, et aussi aura-t-il de l'intérêt pour notre lectorat. Avec un nombre continuellement croissant de journaux à la fois imprimés et en ligne dans lesquels il est possible de publier des documents ayant trait à l'anesthésie, les auteurs disposent d'un très grand nombre d'options à envisager pour l'envoi de leur travail académique. Il y a néanmoins plusieurs principes, apparemment basiques et évidents, qui peuvent arrêter le processus de soumission avant même son démarrage ou peu de temps après la toute première lecture. Ce sont des concepts et détails que tout auteur doit prendre en ligne de compte, qu'il s'agisse de sa «première fois » ou du travail d'un vétéran accompli.

Ces principes de la rédaction scientifique, bien qu'ils ne soient pas destinés à être complets, peuvent être considérés comme un guide sur la façon de voir démarrer le processus de soumission et de révision sur une note positive. Tout d'abord, l'orthographe et la grammaire qui, si elles ne sont pas fondamentales pour l'exactitude et l'importance des faits scientifiques rapportés, n'en sont pas moins très importantes. Si négligés, ils peuvent incommoder les différents réviseurs, diminuant peut-être leur enthousiasme à investir du temps dans le processus de révision et, au pire, altérant leur compréhension d'un message qui devrait être clairement exposé (c'est-à-dire les constatations scientifiques); dans les deux cas, cela peut avoir une répercussion négative sur l'aboutissement de la révision. Il est assez facile de résoudre ce problème compte tenu de la vaste gamme de logiciels et ressources en ligne disponibles pour aider les auteurs. ${ }^{\mathrm{A}}$ De fait, le Journal continue à élargir sa portée internationale: nous encourageons et recevons des soumissions venant d'auteurs qui pourraient faire un bon usage de ces ressources, y compris de ceux pour lesquels l'anglais n'est pas la langue maternelle.

D'autres outils, facilement disponibles, peuvent être envisagés au cours de la rédaction, ${ }^{2}$ mais par-dessus tout, les lignes directrices proposées par le réseau EQUATOR (Enhancing the QUAlity and Transparency Of health Research) sur la façon de rédiger sont facilement accessibles dès les premiers stades de préparation et d'exécution des études. Cette initiative internationale établit ses lignes directrices pour la rédaction (par exemple, les directives CONSORT pour les études randomisées et contrôlées) qui sont facilement disponibles; elles peuvent être utilisées comme « listes de contrôle » utiles, insistant sur les nombreux éléments qui doivent apparaître dans chaque étude et, par conséquent, dans chaque manuscrit. Ces éléments du plan sont effectivement essentiels pour améliorer la communication scientifique, contribuant à assurer sa fiabilité, son exactitude et, finalement, la valeur globale de cette communication. Ils sont également de plus en plus utilisés par les réviseurs pour identifier ce qui doit apparaître dans chaque article, la présence ou l'absence de chacun de ces éléments donnant lieu à une enquête poussée. Dit autrement, s'assurer que ces éléments sont détaillés de façon claire et inclus dans chaque manuscrit est un autre point qui facilite le travail du réviseur et augmente donc les chances de voir la soumission acceptée. Le fait que ces lignes directrices d'EQUATOR doivent, dans l'idéal, être utilisées très en amont de la préparation d'un manuscrit (c'est-à-dire, tôt dans la phase de conception de n'importe quelle étude) pour s'assurer que ces dernières seront menées avec tous les éléments nécessaires pour permettre sa publication est quelque chose de largement sous-estimé.

L'enregistrement d'une étude clinique sur un site d'enregistrement accessible au public (tel que www. clinicaltrials.gov) est un aspect fondamentalement 
important dans la planification, l'exécution et la publication d'une étude. Alors que cette suggestion a été fortement encouragée depuis longtemps dans de nombreux processus de soumission des journaux, cela va bientôt devenir une exigence généralisée. Il ne faut pas sous-estimer l'importance d'écrire et réviser un article dans le contexte qui a été initialement conçu (par exemple, avec des critères d'évaluation définis a priori). C'est important pour les études randomisées contrôlées et aussi pour de nombreux autres documents qui sont soumis, des études observationnelles jusqu'à même des comptes rendus de cas cliniques.

Les problèmes statistiques sont une partie essentielle et reçoivent beaucoup d'attention au cours du processus de révision. En conséquence, la consultation et l'acquisition précoce de ressources statistiques vont loin dans la bonne planification, réalisation et potentielle publication d'une étude, quelle qu'elle soit. Cela est trop souvent ignoré, mais son importance ne peut pas être surestimée. De nombreuses publications sont arrêtées avant même d'atteindre le stade de la première révision à cause de problèmes statistiques. De façon idéale, cette consultation doit commencer aux stades les plus précoces de la planification de l'étude, mais si cela n'a pas été fait, elle doit être incorporée au strict minimum dans la rédaction définitive du manuscrit et sa relecture par l'auteur. Voici un autre exemple où les lignes directrices EQUATOR ont toute leur valeur, avec les lignes directrices $\mathrm{SAMPL}^{\mathrm{B}}$ qui fournissent des conseils pour rapporter les données statistiques. De nombreux journaux ont leurs propres préférences ou matrices pour la présentation des résultats statistiques et les autres questions associées, et les auteurs potentiels ont tout intérêt à bien les étudier avant de faire une soumission.

De fait, chaque journal (et le nôtre ne fait pas exception) a son propre formatage global et unique et des directives régissant les soumissions. Ces directives aux auteurs ${ }^{\mathrm{C}}$ sont facilement accessibles en ligne et devraient être la première ressource référencée et le point de départ de tout auteur potentiel. Même s'il est vrai que la lecture de ces directives est quelque peu ardue et contient souvent des détails plutôt techniques, elle fournit un guide clair ce qui est attendu du Journal au cours de la soumission d'un manuscrit. Il est donc plutôt étonnant que ces directives ne soient que sporadiquement suivies. Quand on demande combien de fois ces instructions ont été regardées et téléchargées chaque année, on s'aperçoit que le chiffre est habituellement très inférieur au nombre total de soumissions que nous recevons. Le respect du formatage du Journal et des instructions est également un point que de nombreux réviseurs prennent en compte et ils peuvent parfois fournir des informations tacites intéressantes sur l'historique d'un manuscrit. Par exemple, si un manuscrit envoyé au Journal $X$ est en fait formaté pour le Journal $Y$, il est alors facile aux réviseurs du Journal $X$ d'en déduire qu'il a été très probablement déjà soumis (et rejeté) ailleurs, remettant très tôt et inutilement en cause le contenu de l'article au cours du processus de révision, ce qui constitue évidemment un net désavantage pour les auteurs.

En résumé, il y a quelques points très simples qui, lorsqu'ils sont respectés très tôt au cours du processus de rédaction et de soumission, peuvent attribuer au manuscrit un crédit optimal en termes de revue positive et d'acceptation ultérieure pour publication. Lire les Directives aux auteurs, vérifier l'orthographe et la grammaire, et faire réviser le travail par un auteur ayant l'anglais comme langue maternelle, impliquer un statisticien à tous les stades, l'utilisation de lignes directrices de publication largement admises et, enfin, mais certainement pas le moindre, enregistrer le projet sur un site d'enregistrement accessible au public avant de commencer.

J'aurai plaisir à continuer de servir comme rédacteur en chef et j'encourage fortement les soumissions d'articles de sciences cliniques et fondamentales de types variés, y compris des rapports sur des recherches originales, des synthèses et, oui aussi, quelquefois des rapports de cas apportant une information unique. $^{3}$ Mon espoir est que cet éditorial apportera quelques conseils préliminaires aux auteurs potentiels ainsi qu'aux investigateurs planifiant un travail qu'ils espèrent voir publier. De même, les réviseurs peuvent l'utiliser pour identifier quels éléments prendre en compte pour leurs propres lectures. La publication de documents scientifiques revus de façon rigoureuse est bénéfique pour nous tous et quand elle est faite correctement, elle peut représenter la meilleure collaboration possible entre des auteurs ayant un message scientifique à transmettre et des journaux ayant un forum permettant la diffusion du message. J'espère rester impliquée dans ce processus gratifiant le plus longtemps possible.

Acknowledgments This submission was handled by Dr. Gregory Bryson, Deputy Editor-in-Chief, Canadian Journal of Anesthesia. Cette soumission a été traitée par Dr Gregory Bryson, rédacteur en chef adjoint, Journal canadien d'anesthésie.

Conflicts of interest None declared.

Conflit d'intérêt Aucun déclaré.

\section{References}

1. Grocott HP. Our Diamond Jubilee: a time to celebrate the past and envision our future. Can J Anesth 2014; 61: 1-4.

2. O'Leary JD, Crawford $M W$. Review article: Reporting guidelines in the biomedical literature. Can J Anesth 2013; 60: 813-21.

3. Plaud B. A new option for the treatment of anaphylaxis linked to steroidal neuromuscular blockers: How much value should we grant to case reports? Can J Anesth 2014; 61: 511-8. 